\title{
Special issue on "Pythagorean fuzzy set and its extensions in decision-making process"
}

\author{
Harish Garg ${ }^{1}$
}

Published online: 23 May 2019

(c) The Author(s) 2019

Multiple attribute decision making (MADM), as one of the most important parts of modern decision-making science, has been widely studied and successfully applied to many fields. Today, however, with the development of society as well as the economy, practical decision-making problems are becoming more and more complex. Thus, it is difficult for a decision maker to make their decisions based on exact or crisp numbers. For this reason, the theory of fuzzy sets has come into the play. Because of the need for better and detailed membership functions, ordinary fuzzy sets have been extended to intuitionistic fuzzy sets, intervalvalued Intuitionistic fuzzy sets, hesitant fuzzy sets, linguistic interval-valued Intuitionistic fuzzy sets, Pythagorean fuzzy sets. Each of these extensions has been used in the solutions of single criterion and multiple criteria decision making problems.

Nowadays, many new extensions of the ordinary or intuitionistic fuzzy sets are proposed and they are expected to be competitive with the other extensions in the future. Among them, Pythagorean fuzzy sets are the generalization of the intuitionistic fuzzy sets and widely applicable in many fields. After its appearance, several extensions such as intervalvalued Pythagorean fuzzy sets, hesitant Pythagorean fuzzy sets, Linguistic Pythagorean fuzzy sets come into the play to solve the decision-making problems. So it is necessary to provide a dedicated forum for discussion on these settings and their extensions, and applications in the real world as well.

This special issue includes twelve papers on decisionmaking theory and applications using Pythagorean fuzzy sets. They have been selected after a peer review process with at least three reviewers per papers.

The first paper titled New similarity measure and distance measure for Pythagorean fuzzy set, authored by Peng, defines

Harish Garg

harishg58iitr@gmail.com

1 School of Mathematics, Thapar Institute of Engineering and Technology, Deemed University, Patiala, Punjab 147004, India a similarity and distance measure for Pythagorean fuzzy sets which depends on four parameters, i.e., $a, b, t$ and $p$, where $p$ is the $L_{p}$ norm and $a, b, t$ identify the level of vagueness. Some properties of the proposed measures are also discussed by them. Further, some counterintuitive examples are presented to state their availability of similarity measure.

The second paper, titled Decision making method based on Pythagorean fuzzy sets and its application to solid waste management by Abdullah and Goh presents a modified decision-making trial and evaluation laboratory (DEMATEL) method characterized by PFS for linguistic variables and applies the proposed method to the case of solid waste management. In it, a linguistic variable of influence, experts' weights and score function are utilized.

The third paper Exponential similarity measures for Pythagorean fuzzy sets and their applications to pattern recognition and decision-making process authored by Nguyen et al. proposes an exponential similarity measure by using the exponential function for the membership and the non-membership degrees for measuring the similarities between two or more objects. Several desirable properties and counter-intuitive cases are given to show the effectiveness of the measures with the existing similarity measures. Problems related to pattern recognition and decision-making problems are presented to demonstrate it.

Akram et al. define two new operations, namely, maximal product and residue product of Pythagorean fuzzy graphs that can allow the mathematical design of a network in their paper titled Certain graphs under Pythagorean fuzzy environment. Also, their work proposes some necessary and sufficient conditions for these two products to be regular.

The fifth paper Simplified interval-valued Pythagorean fuzzy graphs with application, also by Akram et al., presents a concept of a simplified interval-valued Pythagorean fuzzy graph to describe uncertain information in graph theory. Two new operators on it are defined and studied their properties in terms of the degree and the total degree of a vertex.

Pythagorean fuzzy topological spaces by Olgun et al., the sixth paper, extends the notion of fuzzy and the intuitionis- 
tic fuzzy topological space to the notion of the Pythagorean fuzzy topological spaces. Similar to classical function, they defined the Pythagorean fuzzy continuity and characterize the concept. Based on this concept, they give a method to construct a Pythagorean fuzzy topology on a given non-empty set.

Kumar et al. presents an approach for solving the transportation problem by using Pythagorean fuzzy sets in the seventh paper titled A Pythagorean fuzzy approach to the transportation problem whose authors are. A new method is proposed for describing the feasible and optimal solution with Pythagorean fuzzy arithmetic and numerical conditions.

The eight paper Pythagorean fuzzy set and its application in career placements based on academic performance using max-min-max composition by Ejegwa defines a Pythagorean fuzzy relations and max-min-max composition. Some properties of it are investigated. A decision-making approach of career placements on the basis of academic performance is presented using max-min-max composition to ascertain the suitability of careers to applicants.

Hesitant Pythagorean fuzzy interaction aggregation operators and their application in multiple attribute decisionmaking by Weng et al. is the ninth paper. It defines hesitant Pythagorean fuzzy interaction aggregation operators for hesitant Pythagorean fuzzy sets. A new multiple attribute decision-making method is proposed based on the Bonferroni mean interaction averaging and geometric operators under hesitant environment. The applicability of the method is demonstrated through an investment problem.

The tenth paper Multi-granulation Pythagorean fuzzy decision-theoretic rough sets based on inclusion measure and their application in incomplete multi-source information systems whose authors are Mandal and Ranadive presents a multi-granulation Pythagorean fuzzy decisiontheoretic rough sets (MG-PFDTRS) based on Pythagorean fuzzy inclusion measure. A new algorithm for decision making to incomplete multi-source information systems using MG-PFDTRS and the uncertainty measures is proposed. A sensitivity analysis is conducted to examine the mutual funds' investment to examine the robustness of the given decisions.
The eleventh paper Dijkstra algorithm for shortest path problem under interval-valued Pythagorean fuzzy environment whose authors are Enayattabar et al. proposes a method for solving shortest path problems under the interval-valued Pythagorean fuzzy environment. In it, they defined optimality conditions in directed networks to design the solution network and hence present a Dijkstra algorithm to find the cost of interval-valued Pythagorean fuzzy shortest path problems. The applicability of the proposed method is demonstrated with an application of a small sized telecommunication network.

Last but not the least, the twelfth paper AHP-TOPSIS integration extended with Pythagorean fuzzy sets for information security risk analysis by $\mathrm{Ak}$ and Gul proposes a new risk analysis methodology based on AHP-TOPSIS integration. Their aim is to make information security risk analysis comprehensive, efficient and effective with MADM methods by the integration of fuzzy logic. The feasibility and practicality of the proposed approach are given through a case study for information security risk analysis in corrugated cardboard sector.

I hope this issue will provide a useful resource of ideas, techniques, and methods for the research on the theory and applications of Pythagorean fuzzy sets and the decisionmaking problems. I thank all the authors whose contributions and efforts made the publication of this issue possible. I am also grateful to the referees for their valuable and highly appreciated works contributed to select the high quality of papers published in this issue. Finally, my sincere thanks go to Prof. Yaochu Jin, Editor-in-Chief, for his support throughout the process of editing this issue.

Open Access This article is distributed under the terms of the Creative Commons Attribution 4.0 International License (http://creativecomm ons.org/licenses/by/4.0/), which permits unrestricted use, distribution, and reproduction in any medium, provided you give appropriate credit to the original author(s) and the source, provide a link to the Creative Commons license, and indicate if changes were made.

Publisher's Note Springer Nature remains neutral with regard to jurisdictional claims in published maps and institutional affiliations. 